\title{
The convergent procedure versus catheter ablation alone in longstanding persistent atrial fibrillation: a single centre, propensity-matched cohort study
}

\author{
E Maclean ${ }^{1,2}$ MRCP, J Yap ${ }^{1}$ MD, FRCS, B Saberwal ${ }^{1}$ MRCP, S Kolvekar ${ }^{1}$ MS, \\ FRCS, W Lim ${ }^{1}$ MRCP, N Wijesuriya ${ }^{1}$ MRCP, N Papageorgiou ${ }^{1}$ MD, PhD, G \\ Dhillon ${ }^{1}$ MRCP, RJ Hunter ${ }^{1,2}$ FRCP, PhD, FHRS, M Lowe ${ }^{1}$ FRCP, PhD, P \\ Lambiase $^{1}$ FRCP, PhD, A Chow ${ }^{1}$ FRCP, MD, H Abbas ${ }^{1}$ MSc, R Schilling ${ }^{1,2}$ \\ FRCP, MD, E Rowland ${ }^{1}$ FRCP, S Ahsan ${ }^{1}$ FRCP, MD \\ Affiliations: \\ 1. Barts Heart Centre, St Bartholomew's Hospital, W Smithfield, London, EC1A 7BE, UK; This author takes \\ responsibility for all aspects of the reliability and freedom from bias of the data presented and their discussed \\ interpretation \\ 2. William Harvey Research Institute, Charterhouse Square, Barts and the London School of Medicine and \\ Dentistry, Queen Mary University of London, London EC1M 6BQ; This author takes responsibility for all \\ aspects of the reliability and freedom from bias of the data presented and their discussed interpretation
}

\author{
Corresponding author: \\ Dr. Syed Ahsan \\ Barts Heart Centre \\ St Bartholomew's Hospital \\ W Smithfield \\ London \\ EC1A 7BE \\ UK \\ Tel: +44(0)2073 777000 \\ syedahsan@nhs.net
}

\section{KEY WORDS}

Persistent atrial fibrillation

Hybrid surgical ablation

Convergent procedure

Catheter ablation

\section{DISCLOSURES}

Dr Ahsan has received an educational grant and speaker fees from Atricure 


\section{ABSTRACT}

BACKGROUND: Maintenance of sinus rhythm is challenging in patients with longstanding persistent atrial fibrillation $(\mathrm{PeAF})$. Minimally invasive surgical AF ablation may improve outcomes when combined with catheter ablation (the 'convergent' procedure). This study evaluates the safety and efficacy of the convergent procedure versus catheter ablation alone in longstanding PeAF.

METHODS: 43 consecutive patients with longstanding PeAF underwent subxiphoid endoscopic ablation of the posterior left atrium followed by catheter ablation from 2013-2018. The primary outcome was AF-free survival at 12 months; secondary outcomes included change in EHRA class, echocardiographic data, procedural complications, freedom from anti-arrhythmic drugs (AADs), and long term arrhythmia-free survival. Outcomes were compared with a matched group of 43 patients who underwent catheter ablation alone. Both groups underwent multiple catheter ablations as required. Baseline characteristics were similar between groups.

RESULTS: After 12 months, the convergent procedure was associated with increased AF-free survival on AADs (60.5\% versus 25.6\%, $\mathrm{p}=0.002)$ and off AADs (37.2\% versus 13.9\%, $\mathrm{p}=0.025)$, versus catheter ablation. Allowing for multiple procedures, after 30.5 \pm 13.3 months' follow-up the convergent procedure was associated with increased arrhythmia-free survival on AADs $(58.1 \%$ versus $30.2 \%$, $\mathrm{p}=0.016)$ and off AADs (32.5\% versus $11.6 \%, \mathrm{p}=0.036)$ versus catheter ablation. There were more complications in the convergent procedure group (11.6\% versus $2.3 \%, \mathrm{p}=0.2)$. Multivariate analysis identified only the convergent procedure (OR $3.06(1.23-7.6), \mathrm{p}=0.017)$ as predictive of arrhythmiafree survival long term.

CONCLUSIONS: In longstanding PeAF, the convergent procedure is associated with improved arrhythmia-free survival versus catheter ablation alone. Complication rates are significant but have been shown to depreciate with experience.

\section{ABBREVIATIONS}

Antiarrhythmic drugs, AAD; Atrial Fibrillation, AF; Complex Fractioned Electrograms, CFE; European Heart Rhythm Association, EHRA; Left Atrial Diameter, LAD; Left Ventricular Ejection Fraction, LVEF; New York Heart Association, NYHA; Persistent Atrial Fibrillation, PeAF;

80 Pulmonary Vein Isolation, PVI 
83 The morbidity and mortality of Atrial Fibrillation (AF) is well-recognised; approximately $10 \%$ of people over 65 years old are affected by this condition (1). The risk of stroke and heart failure is significantly higher in affected individuals, and rises with increasing age and co-morbidities (2). Catheter ablation of $\mathrm{AF}$ is an established treatment for patients in which sinus rhythm is desirable, such as those with refractory symptoms despite maximal medical therapy, heart failure secondary to $\mathrm{AF}$, or an intolerance to anti-arrhythmic drugs (AADs). Pulmonary vein isolation (PVI) is the cornerstone of catheter ablation, and whilst outcomes are generally regarded as favourable in cases of paroxysmal (< 7 days of continuous AF) (3) and more recently persistent AF ( $>7$ days but continuous duration $<1$ year) (4) (5), there remains a need for a more efficacious rhythm control procedure in patients with longstanding persistent $\mathrm{AF}$ (continuous $\mathrm{AF}>1$ year). A recent 5 year outcome analysis of the BELIEF trial found that patients with longstanding persistent AF who underwent PVI plus additional linear catheter ablation - including superior vena cava (SVC) isolation, septal and roof lines - had a single procedure success rate of $19 \%$ (6).

Recurrence of arrhythmia following catheter ablation for longstanding persistent AF may be mediated by additional arrhythmia drivers outside the pulmonary veins. The posterior left atrial wall in particular harbours rotors, focal drivers, complex fractionated electrograms (CFEs) and a significant concentration of ganglionated plexi in adjacent epicardial fat, and targeted ablation of these substrates has been associated with improved maintenance of sinus rhythm (7-11). In addition, the mismatch in endocardial and epicardial ablation (12-13). However, extensive catheter ablation of the posterior wall is limited by the proximity of other important structures, particularly the oesophagus, and randomised trials such as STAR-AF 2, BOCA and CHASE-AF have not shown incremental benefit of extensive posterior wall ablation over PVI alone (4, 14-15). 
107 Alternatives to catheter ablation include open surgical ablation, which can be performed concomitant 108 with other operations or as a standalone procedure (16). The gold standard of surgical AF ablation 109 the Cox-Maze procedure - has been meta-analysed in patients with longstanding persistent AF and 110 was found to have satisfactory outcomes, with 55\% of patients in sinus rhythm at 12 months (17).

111 Whilst these results are encouraging, uptake of surgical AF ablation as a standalone procedure is 112 limited, possibly by concerns over its relatively invasive nature; of the 91,801 surgical AF ablations performed in the US from 2005-2010, only 5.3\% were standalone (18).

\section{Hybrid ablation}

115 Endoscopic, subxiphoid surgical ablation may deliver much of the advantages of open surgical ablation - extensive posterior wall and pulmonary vein ablation with direct visualisation of other important structures such as the oesophagus and phrenic nerve - but with minimal surgical trauma, standard ventilation and without the need for cardiopulmonary bypass. To achieve a hybrid of epiand endocardial ablation known as the 'convergent' procedure, catheter ablation can be performed either contemporaneously or in a staged procedure (see supplementary figure a). Both single-setting and staged approaches have theoretical advantages; a single procedure is more convenient for patients and provides the surgeon with additional feedback from live endocardial 3D mapping, whilst a staged procedure allows the electrophysiologist to assess more matured surgical lesions, and then optimise them accordingly.

A meta-analysis of 16 studies by Jiang et al. (2018) included 785 patients - 63\% with longstanding persistent $\mathrm{AF}$ - who underwent the convergent procedure and found a pooled arrhythmia-free survival of $73 \%$ off AADs, rising to $83 \%$ allowing for AADs and/or repeat catheter ablations (19). Those individuals undergoing staged procedures had superior success rates when compared with the singlesetting technique, and also demonstrated a non-significant tendency towards fewer complications ( $2 \%$ versus $5 \%$ ). 
133 We aimed to evaluate the safety and long term efficacy of the convergent procedure in a cohort of 134 consecutive patients with longstanding persistent atrial fibrillation, and to compare outcomes with a 135 group of matched controls undergoing catheter ablation alone.

\section{METHODS}

\section{Recruitment}

138 From 2013-2018 at a single UK centre, patients with persistent AF over 12 months' duration were referred for the convergent procedure at the recommendation of their Cardiologist in line with international consensus guidelines. Only patients with a history of previous cardiac surgery,

141 abdominal surgery, or a contraindication to anticoagulation were excluded.

\section{Ethics}

143 All patients provided informed consent. This retrospective analysis was registered with our institution's Clinical Effectiveness Unit; the need for formal ethical approval was waived.

Patient parameters

146 Baseline characteristics were established from electronic records, clinic documentation, investigation results and procedure reports. Duration of AF was discerned from the initial referral letter. All patients underwent pre-procedural transthoracic echocardiogram to determine left atrial size (long axis left atrial diameter - LAD) and left ventricular ejection fraction (Simpson's biplane or visual estimate -

150 LVEF). In line with established definitions, EHRA symptomatic class was determined in all patients,

151 and NYHA class was determined in those with systolic dysfunction (LVEF <50\%) (16).

\section{Convergent procedure}

153 All patients underwent a staged procedure, with the surgical (epicardial) component performed approximately 6 weeks prior to catheter ablation. 
156 Before the epicardial ablation, patients had their anticoagulation stopped for five days (vitamin K

157 antagonist) or two days (direct oral anticoagulant) and then restarted five days subsequently (or longer

158 in the event of complications). All patients received general anaesthesia with standard ventilation, and

159 had an oesophageal temperature probe in situ. In the majority of cases, a subxiphoid incision was

160 made and a transdiaphragmatic approach used with a laparoscope advanced following carbon dioxide

161 insufflation of the abdomen. In the last three patients to be included in our study - in response to a

162 patient returning with a pericardial hernia - the access technique was changed so that the xiphoid

163 process was excised, obviating the need for diaphragmatic incision. Under direct visualisation, the

164 posterior pericardium was then incised and cannulated giving access to the posterior left atrium. The

165 Atricure (OH, USA) EPIsense coagulation catheter with VisiTrax@ was used to produce continuous,

166 intersecting linear lesions across the posterior wall of the left atrium. With the lesion set complete, a

167 pericardial drain was inserted and the incision closed. Direct current cardioversion (DCCV) was

performed to achieve sinus rhythm if required. Patients recovered in the high dependency unit with a view to discharge after 3 days.

Subsequent catheter ablation was performed approximately 6 weeks later under moderate sedation or general anaesthesia and without interruption of antiarrhythmic drugs. Procedures were guided by

CARTO 3-D mapping (Biosense Webster Inc, Diamond Bar, CA). Following trans-septal puncture, heparin was administered to maintain an activated clotting time of 300-400 seconds. Radiofrequency energy was delivered via an irrigated catheter, with power limited to $35 \mathrm{~W}$ and temperature limited to $50^{\circ} \mathrm{C}$. The ablation strategy was operator dependent, but involved (in all cases) completing PVI and delivering additional lesions to achieve posterior wall isolation, additional CFE ablation (time spent was operator dependent) and finally induction, mapping and ablation of any atrial tachycardia. Additional lines were performed at the operators' discretion. In those cases where sinus rhythm was not restored, DCCV was performed. In the absence of complications, patients were discharged the following day. 
183 In line with consensus guidelines, in both groups a 3 month blanking period was observed starting from the date of catheter ablation (20). For patients undergoing the convergent procedure, clinical review took place at 3 months (with ECG), 6 months (with 72 hour holter analysis) and 12 months (with echocardiogram, ECG and symptom-guided 72 hour holter monitor). For patients undergoing catheter ablation alone, clinical review took place at 3 months (with ECG or symptom-guided holter), and, unless a recurrence of symptoms prompted earlier review, patients were reviewed again at 12 months (with echocardiogram, ECG or symptom-guided 72 hour holter monitor). Patients with pacemakers in situ underwent device interrogation in addition to holter monitoring. Further follow-up took place annually or sooner if clinically indicated. 30 seconds of documented AF outside of the blanking period was considered a recurrence. The presence of any other atrial arrhythmia was also recorded. AAD use was assessed at 12 months, and anticoagulation was continued as indicated by $\mathrm{CHA}_{2} \mathrm{DS}_{2} \mathrm{VASc}$ score. The primary outcome was AF-free survival at 12 months. Secondary outcomes included incidence of atrial tachycardia, change in NYHA and EHRA class, procedural complications, echocardiographic data, freedom from AADs, and arrhythmia-free survival long term. In both groups, patients underwent additional DCCV or repeat catheter ablation as indicated; in these cases, a further 3 month blanking period was observed and follow-up restarted.

\section{Statistical analysis}

Statistical analysis was performed using R and SPSS (v. 26, IBM Corporation). Categorical group parameters were compared using Z-tests. Normally distributed data are presented as mean \pm standard deviation and non-normally distributed data as median (interquartile range). Continuous parameters were analysed using two-tailed unpaired t tests for normally distributed data or the Mann-Whitney U test for non-normally distributed data. For grouped outcomes, dichotomous data were compared using Fisher's exact and continuous data were compared with analysis of covariance (ANCOVA) using preintervention data as a covariate. Kaplan-Meier plots were generated and the survival distributions compared using the log rank test. Univariate logistic regression analysis was performed for all 
covariates with odds ratios for the primary outcome provided with $95 \%$ confidence intervals.

210 Significant covariates were entered into a multivariate model. The level of significance for all tests

211 was set at $\mathrm{p}<0.05$.

212

213 Matched control group

214 A pool of 312 patients with longstanding persistent AF who had undergone at least one radiofrequency catheter ablation at the same institution from 2013-2018 was identified. One-to-one propensity matching was performed via a greedy (nearest neighbour) model, scoring the covariates of age, gender, ejection fraction, atrial size, use of class I or class III AADs, number of previous ablations (prior to 2013) and duration of persistent AF. In line with Austin's 2011 recommendations for eliminating confounder bias, a pre-specified target calliper distance equal to 0.2 pooled standard deviations of the logit of the propensity score was used (21). Control patients were identified for all cases, with all but 3 individuals matching inside these restrictions.

\section{RESULTS}

\section{Participants}

227

43 consecutive patients underwent both components of the convergent procedure. Baseline parameters are shown in table 1 alongside 43 matched controls; characteristics were similar between groups. 
Table 1: Baseline characteristics for patients undergoing the convergent procedure ('Convergent

Procedure') versus matched controls undergoing catheter ablation alone ('Catheter Ablation Alone') -

significant $\mathrm{p}$ values in bold

\begin{tabular}{|c|c|c|c|}
\hline Parameter & $\begin{array}{l}\text { Convergent } \\
\text { Procedure } \\
(n=43)\end{array}$ & $\begin{array}{l}\text { Catheter } \\
\text { Ablation Alone } \\
\qquad(n=43)\end{array}$ & p \\
\hline Age & 68.6 years $( \pm 7.7)$ & 65.5 years $( \pm 7.5)$ & 0.096 \\
\hline Male & $74.4 \%(n=32)$ & $74.4 \%(n=32)$ & 1 \\
\hline Duration of AF & 36 months (30) & 30 months (28) & 0.19 \\
\hline Left atrial diameter & $47.4 \mathrm{~mm}( \pm 6.3)$ & $47.5 \mathrm{~mm}( \pm 7.4)$ & 0.84 \\
\hline LVEF & $50 \%$ & $50 \%(20)$ & 0.77 \\
\hline Class III AAD & $51.2 \%(n=22)$ & $48.8 \%(n=21)$ & 0.83 \\
\hline Class I AAD & $9.3 \%(n=4)$ & $11.6 \%(n=5)$ & 0.73 \\
\hline Beta blocker & $95.3 \%(n=41)$ & $97.7 \%(n=42)$ & 0.56 \\
\hline Previous ablation & $34.8 \%(n=15)$ & $34.8 \%(n=15)$ & 1 \\
\hline Previous DCCV & $65.2 \%(n=28)$ & $51.2 \%(n=22)$ & 0.27 \\
\hline PPM & $4.6 \%(n=2)$ & $0 \%(n=0)$ & 0.49 \\
\hline $\begin{array}{l}\text { Hypertrophic/Dilated } \\
\text { Cardiomyopathy }\end{array}$ & $11.6 \%(n=5)$ & $2.3 \%(n=1)$ & 0.2 \\
\hline $\begin{array}{l}\text { Warfarin (remainder } \\
\text { DOAC) }\end{array}$ & $60.5 \%(n=26)$ & $39.5 \%(n=17)$ & 0.08 \\
\hline EHRA class & $2.6( \pm 0.7)$ & $2.7( \pm 0.8)$ & 0.44 \\
\hline NYHA class & $2.5( \pm 0.5)$ & $2.4( \pm 0.5)$ & 0.65 \\
\hline
\end{tabular}




\section{Rhythm control procedures}

247 Full lesion set data is available as a supplementary file (supplementary table a). Catheter ablation was performed by a Consultant Electrophysiologist with a minimum of 5 years' experience in AF ablation. As the convergent procedure was performed via a staged approach, the convergent group underwent 43 surgical procedures and subsequently 60 catheter procedures, whereas the control group underwent 55 catheter procedures ( 103 versus $55, \mathrm{p}<0.001$ ). The catheter lesion sets used were similar between groups; all participants had catheter pulmonary vein isolation, $69.7 \%$ had cavotricuspid isthmus lines, 45.3\% had CFE ablation, 32.6\% had roof lines, $11.6 \%$ had mitral lines, and $22.6 \%$ had other ablation lines. The need for follow-up DC cardioversion was similar between groups (25.6\% of convergent patients versus $13.9 \%$ of controls, $\mathrm{p}=0.28$ ) and the need for repeat catheter ablation was also similar (32.6\% of convergent patients versus $23.3 \%$ of controls, $\mathrm{p}=0.47$ ).

\section{Outcomes}

Outcome data is shown in table 2, with symptomatic and echocardiographic data shown in table 3.12 months following the index procedure, the convergent procedure was associated with increased AFfree survival on AADs (60.5\% versus $25.6 \%, \mathrm{p}=0.002)$ and off AADs (37.2\% versus $13.9 \%, \mathrm{p}=0.025)$ when compared with catheter ablation. Allowing for multiple procedures, after a mean follow-up of $30.5 \pm 13.3$ months the convergent procedure was associated with increased arrhythmia-free survival long term on AADs (58.1\% versus $30.2 \%, \mathrm{p}=0.017)$ and off $\mathrm{AADs}(32.5 \%$ versus $11.6 \%, \mathrm{p}=0.036)$ when compared with catheter ablation. There was significant symptomatic improvement in both groups, and no significant change in echocardiogram findings (table 3). Survival distribution is shown 
in figure 1; by log rank test the probability of long term arrhythmia-free survival was significantly higher in the convergent group ( $\mathrm{p}=0.003)$. There was no difference in freedom from AADs between groups, and there was a tendency towards new atrial tachycardias in the convergent group.

Table 2: Outcome data for patients undergoing the convergent procedure versus catheter ablation alone - significant $\mathrm{p}$ values in bold

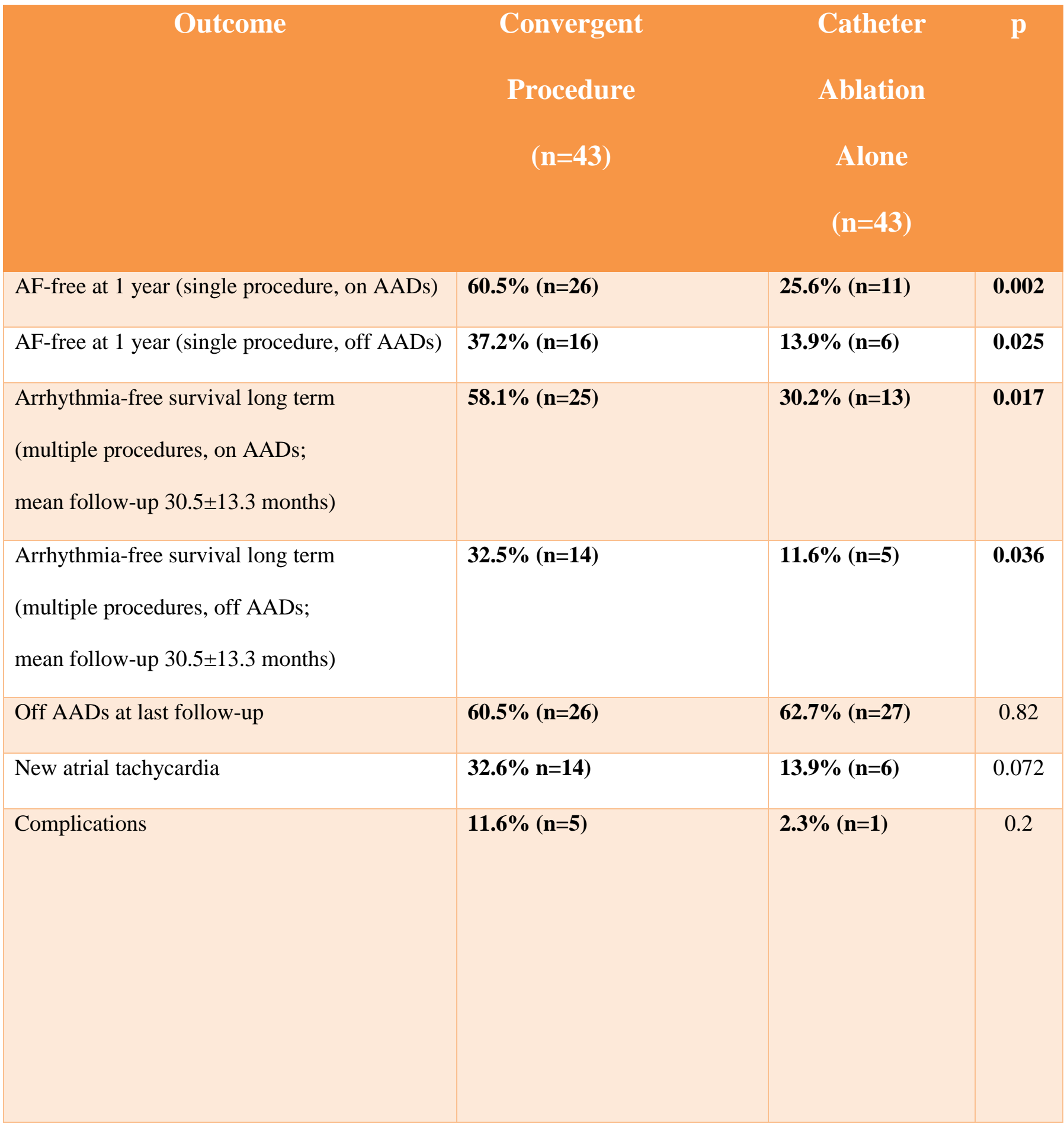




\begin{tabular}{|c|c|c|c|}
\hline $\begin{array}{c}\text { Tamponade } \\
\text { (pericardiocentesis) }\end{array}$ & $\mathbf{2}$ & $\begin{array}{c}\text { Tamponade } \\
\text { (pericardiocentesis) }\end{array}$ & $\mathbf{1}$ \\
\hline $\begin{array}{c}\text { Emergency } \\
\text { sternotomy }\end{array}$ & $\mathbf{1}$ & \\
\hline Pericardial hernia & $\mathbf{1}$ & \\
\hline Phrenic nerve palsy & $\mathbf{1}$ & \\
\hline
\end{tabular}

Table 3: Symptomatic and echocardiographic data for patients undergoing the convergent procedure versus catheter ablation alone - significant $p$ values in bold

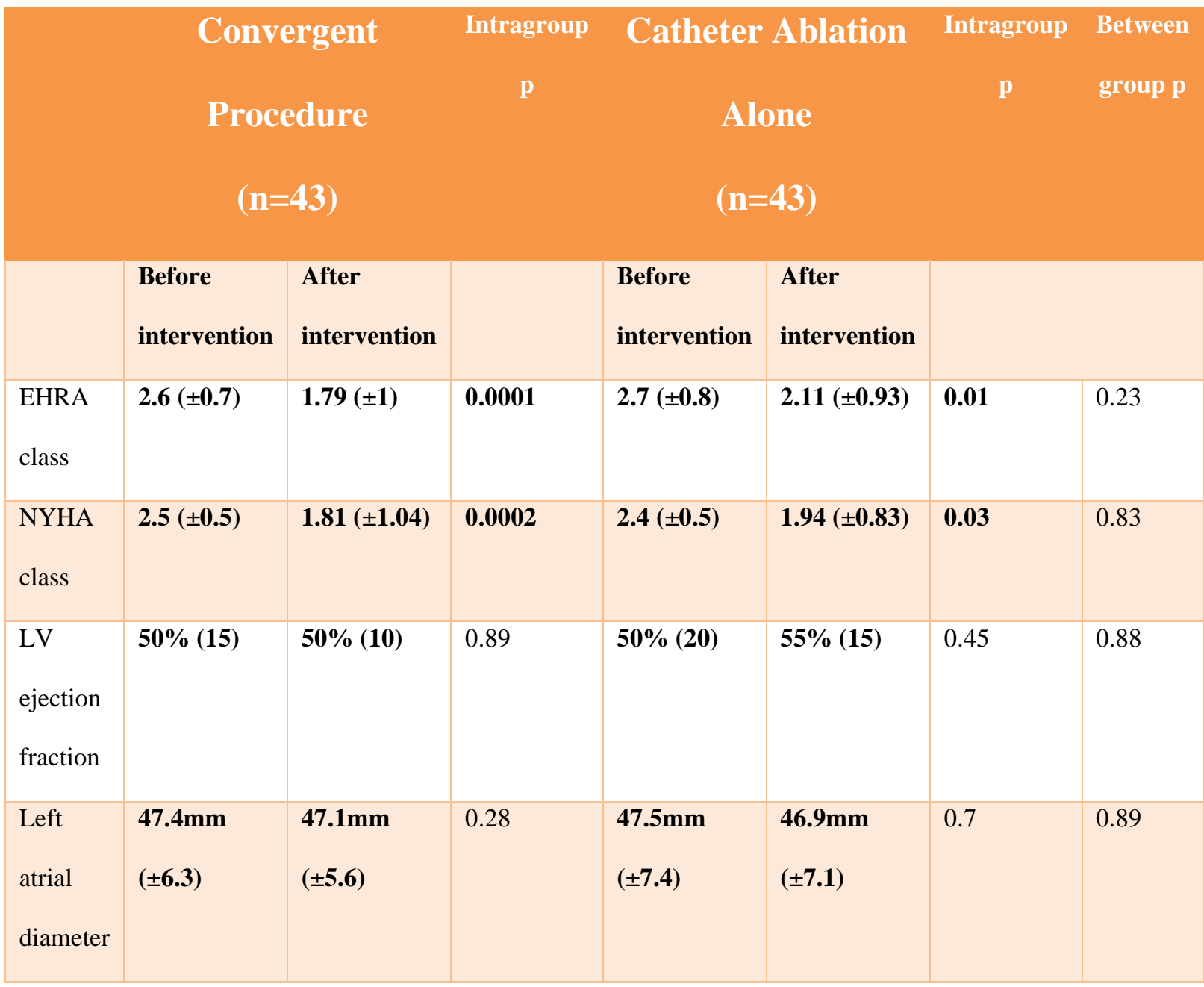


Univariate analysis of risk factors, echocardiographic data and treatment received was performed with odds ratios $(95 \% \mathrm{CI})$ for arrhythmia-free survival; significant parameters were passed into a multivariate analysis (see supplementary figure b and supplementary table b). By univariate analysis, only the convergent procedure (OR $4.13(1.49-11.39), \mathrm{p}=0.006)$ and history of previous catheter ablation (OR 4.05 (1.39-11.76), $\mathrm{p}=0.01)$ were associated with arrhythmia-free survival. Adjusting for history of previous catheter ablation, multivariate analysis demonstrated an association of the convergent procedure with arrhythmia-free survival (OR 3.06 (1.23-7.59), $\mathrm{p}=0.017$ ).

\section{Complications}

There were more complications in the convergent group although this did not reach statistical significance $(11.6 \%$ versus $2.3 \%, \mathrm{p}=0.2)$. With regards to the epicardial procedure, one patient suffered an inferior vena cava rupture requiring emergency sternotomy, and another patient presented six months post-operatively with a pericardial hernia which required surgical correction. During catheter ablation, two patients from the convergent group and one control patient developed tamponade requiring emergency pericardiocentesis. A patient in the convergent group also developed a phrenic nerve palsy following catheter ablation. No strokes or deaths were observed in either group.

\section{DISCUSSION}

Our propensity-matched cohort study suggests that, in patients with longstanding persistent AF, the convergent procedure is associated with increased freedom from AF at one year - and improved arrhythmia-free survival long term - versus catheter ablation alone. The two groups had similar 
baseline characteristics and underwent similar catheter ablation protocols and, as such, we suggest the difference in outcomes between patients is likely to have been mediated by the extensive ablation of the posterior left atrium that takes place during the convergent procedure.

Our finding that $58.1 \%$ of patients who underwent the convergent procedure were arrhythmia-free long-term (with over one third still taking AADs) is inferior to the $73 \%$ success rate reported in Jiang's 2018 meta-analysis (19). We suggest that this discrepancy is mediated by the marked electroanatomic heterogeneity of our cohort, which included patients with cardiomyopathy, severe LV systolic dysfunction, pacemakers and, in over a third of cases, a history of prior unsuccessful rhythm control. This may also account for the lower than expected efficacy of catheter ablation alone; allowing for AADs, only $30 \%$ of our control group patients were in sinus rhythm at last follow-up despite repeat procedures.

There was a tendency towards increased complications in the convergent group, and our overall complication rate was considerable. This may in part reflect a high risk population often with very longstanding AF, severe atrial dilatation and significant co-morbidities, together with the initial learning curve associated with a new technique. Similar studies have reported comparable complication rates. When Kulbak et al. (2015) described their initial experience with the convergent procedure in 28 patients, they reported a 10.7\% incidence of complications (22). By 2018, the same group reported results from a larger cohort $(n=100)$ with a revised complication rate of $3 \%$, suggesting that complications attenuate with operator experience (23). Likewise, Bulava's 2015 study of 50 patients who underwent the convergent procedure reported a $13.7 \%$ complication rate, all of which occurred in the first 15 patients; complications subsequently ceased after the authors modified their surgical technique (24). Accordingly, by adapting our incision to include resection of the xiphoid process rather than a transdiaphragmatic approach, our operators have abrogated the incidence of future pericardial hernias in particular.

The convergent group had a tendency towards an increased incidence of atrial tachycardias, which were observed in $32.6 \%(n=14)$ of patients undergoing epicardial ablation. None of these originated from the posterior wall, and one was localised to the right upper pulmonary vein. The remainder were dependent on the cavotricuspid isthmus $(n=5)$, mitral isthmus $(n=3)$, the anterior wall $(n=3)$, or the 
appendage ridge $(\mathrm{n}=2)$. As such, investigators may wish to consider empirical CTI lines in all patients undergoing the convergent procedure.

\section{Limitations}

Our study has important limitations. Our arrhythmia monitoring strategy included repeated ECG and holter analysis; this may not have detected short-lived asymptomatic atrial arrhythmias outside of the blanking period. In addition, many control group patients did not receive a 6 month review, and as such asymptomatic patients may not have had a recurrence of arrhythmia identified until 12 months post-procedure. Our data is non-randomised and, whilst the propensity score matching process generated a control group with similar baseline characteristics, there may have been unobserved variables which affected outcomes. The nature of our procedures precluded blinding of the operators or patients. As such, operators were aware if patients had undergone epicardial ablation, which may in turn have biased their catheter ablation or AAD strategies. Likewise, the symptomatic benefit seen in the convergent group does not control for the additive placebo effect that may stem from undergoing a novel and ostensibly more rigorous surgical procedure.

\section{Conclusion}

In a challenging cohort of patients with refractory, longstanding persistent AF, our study suggests that the convergent procedure is associated with increased arrhythmia-free survival versus catheter ablation alone. The results of randomised controlled trials - such as DEEP-AF and CONVERGE - are now required to further clarify the safety and efficacy of hybrid ablation techniques.

\section{REFERENCES}

1. Lloyd-Jones DM. Cardiovascular health and protection against CVD: more than the sum of the parts? Circulation, 2014; 104: 1671-1673.

2. Gage BF, Waterman AD, Shannon W et al. Validation of clinical classification schemes for predicting stroke: results from the National Registry of Atrial Fibrillation. JAMA. 2001; 285(22):2864-70.

3. Kuck KH, Fürnkranz A, Chun KR et al. Cryoballoon or radiofrequency ablation for symptomatic 
paroxysmal atrial fibrillation: reintervention, rehospitalization, and quality-of-life outcomes in the FIRE AND ICE trial. Eur Heart J. 2016;37(38):2858-2865.

4. Verma A, Jiang CY, Betts TR et al. Approaches to catheter ablation for persistent atrial fibrillation. N Engl J Med. 2015; 372(19):1812-22

5. Koektuerk B, Yorgun H, Hengeoez O et al. Cryoballoon Ablation for Pulmonary Vein Isolation in Patients With Persistent Atrial Fibrillation: OneYear Outcome Using Second Generation Cryoballoon. Circ Arrhythm Electrophysiol. 2015 (5):10739

6. Mohanty S, Di Biase L, Trivedi C et al. Significance of left atrial appendage isolation in patients with long-standing persistent atrial fibrillation undergoing catheter ablation. European Heart Journal, 2018; 39 (supp. 1) ehy564.364

7. Roberts-Thomson KC, Stevenson I, Kistler PM et al.

The role of chronic atrial stretch and atrial fibrillation on posterior left atrial wall conduction. Heart Rhythm. 2009;6(8):1109-17

8. Tilz RR, Rillig A, Thum AM et al. Catheter ablation of long-standing persistent atrial fibrillation: 5-year outcomes of the Hamburg Sequentil Ablation Strategy. J Am Coll Cardiol. 2012. 60(19):1921-9

9. Baykaner T, Lalani GG, Schricker A et al. Mapping and ablating stable sources for atrial fibrillation: summary of the literature on Focal Impulse and Rotor Modulation (FIRM). J Interv Card Electrophysiol. 2014;40:237-244.

10. Narayan SM, Krummen DE, Clopton P et al. Direct or coincidental elimination of stable rotors or focal sources may explain successful atrial fibrillation ablation: on treatment analysis of the CONFIRM (CONventional ablation for AF with or without Focal Impulse and Rotor Modulation) Trial. J Am Coll Cardiol. 2013;62:138-147.

11. Pokushalov E, Romanov A, Katritsis D et al. Ganglionated plexi ablation vs linear ablation in patients undergoing pulmonary vein isolation for persistent/longstanding persistent atrial fibrillation: a randomized comparison. Heart Rhythm. 2013;10:1280-1286.

12. Yang F. Converging Towards an Effective Cure for Persistent AF: A Review of Techniques and the case for a First-line Multi-disciplinary approach. EP Lab Digest 2015, 15 (5)

13. Burstein $\mathrm{B}$, Comtois $\mathrm{P}$, Michael $\mathrm{G}$ et al. Changes in connexin expression and the atrial fibrillation substrate in congestive heart failure. Circ Res. 2009;105:1213-1222.

14. Wong, K. C., Paisey, J. R., Sopher, M. et al. No benefit of complex fractionated atrial electrogram ablation in addition to circumferential pulmonary vein ablation and linear ablation: benefit of complex ablation study. Circ. Arrhythm. Electrophysiol. 8, 1316-1324

15. Vogler J, Willems S, Sultan A et al. Pulmonary Vein Isolation Versus Defragmentation: The CHASE-AF Clinical Trial. J Am Coll Cardiol. 2015 Dec 22;66(24):2743-2752

16. Cox JL, Schuessler RB, D'Agostino HJ Jr et al. The surgical treatment of atrial fibrillation. III. Development of a definitive surgical procedure. J Thorac Cardiovasc Surg. 1991;101(4):569-83

17. Wang $\mathrm{X}$, Wang $\mathrm{C}$, Ye $\mathrm{M}$ et al. Left atrial concomitant surgical ablation for treatment of atrial fibrillation in cardiac surgery: A meta-analysis of randomized controlled trials PLoS 
408 18. Ad N, Suri RM, Gammie JS et al. Surgical ablation of atrial

409 fibrillation trends and outcomes in North America. J Thorac Cardiovasc Surg. 2012;144(5):1051-60.

410 19. Jiang YQ, Tian Y, Zeng LJ et al. The safety and efficacy of hybrid ablation for

411 the treatment of atrial fibrillation: A meta-analysis. PLoS One. 2018 13(1):e0190170

412 20. Calkins H, Hindricks G, Cappato R et al.

$4132017 \mathrm{HRS} / \mathrm{EHRA} / \mathrm{ECAS} / \mathrm{APHRS} / \mathrm{SOLAECE}$ expert consensus statement on catheter and surgical

414 ablation of atrial fibrillation Heart Rhythm. 2017;14(10):e275-e444.

21. Austin PC. Optimal caliper widths for propensity-score matching when estimating differences in means and differences in proportions in observational studies Pharm Stat. 2011;10(2):150-61

417 22. Kulbak G, Greene M, Chen O et al. Initial Experience with the Convergent Atrial Fibrillation

418 Ablation Procedure International Society for Minimally Invasive Cardiothoracic Surgery 2015, Berlin

419 23. Yang F, Miller A, Saxena A et al. Initial experience with the convergent atrial fibrillation abltion 420 procedure: the first 100 patients Heart Rhythm 2018, 15(5), e590 


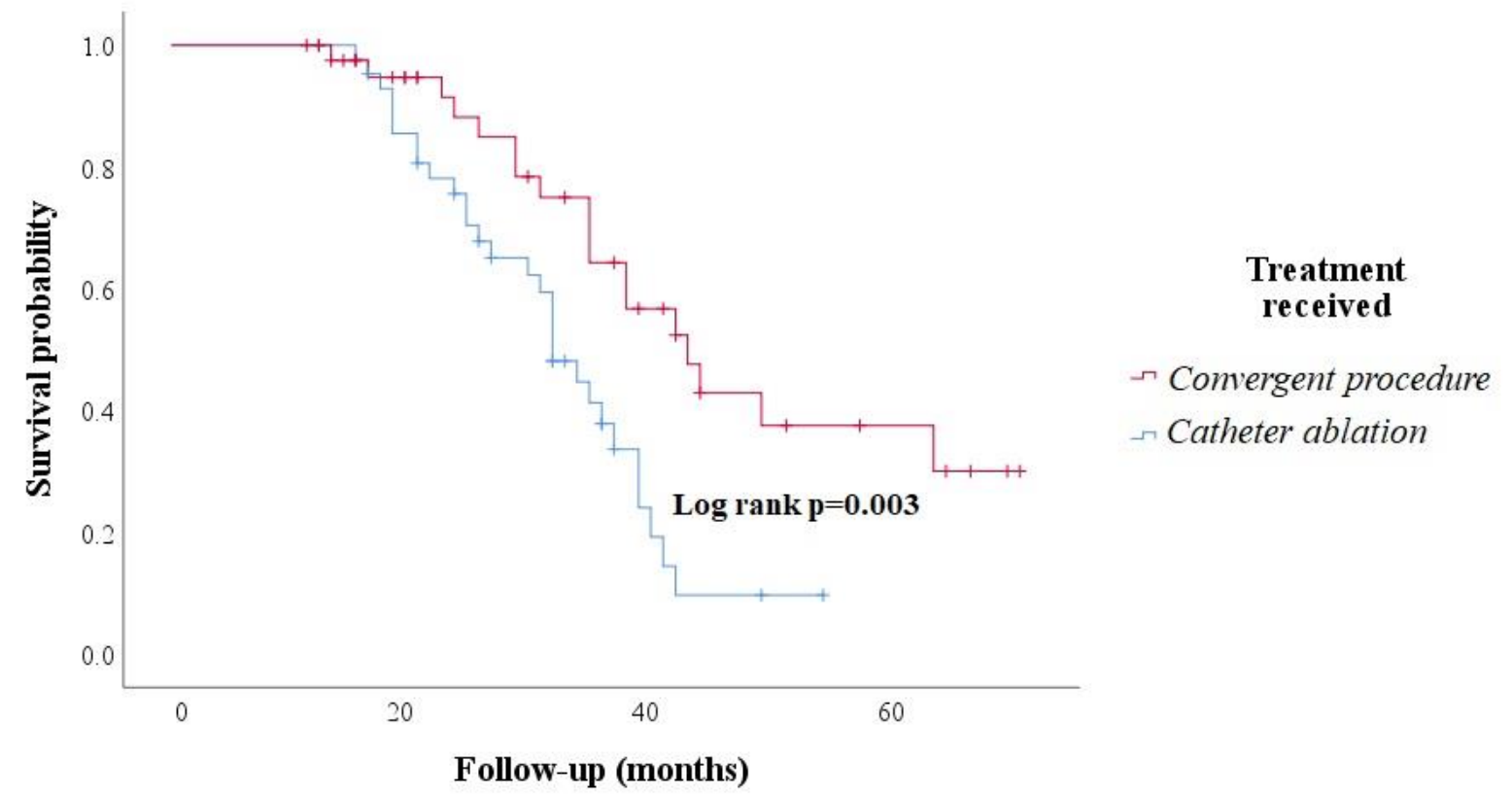

Figure 1: Kaplan Meier plot showing long term arrhythmia-free survival probability for patients undergoing the convergent procedure versus catheter ablation only (allowing for AADs; mean followup $30.5 \pm 13.3$ months) 
489

$490 \quad$ Figure 1: Kaplan Meier plot showing long term arrhythmia-free survival probability for patients 491 undergoing the convergent procedure versus catheter ablation only (allowing for AADs; mean follow492 up $30.5 \pm 13.3$ months)

493

494 Supplementary figure a: Diagram of the lesion set achieved during the epicardial and endocardial 495 components of the convergent procedure (credit: Yang F 2015 (12))

496

497 Supplementary figure b: Odds ratios for long term arrhythmia-free survival according to treatment 498 received and patient parameters

499

500

501

502

503

504

505 